\title{
RECYCLING CIRCLE PLANES
}

\author{
Burkard Polster
}

\begin{abstract}
The main aim of this paper is to introduce new ways of constructing flat projective planes from spherical circle planes and cylinder circle planes. We shall also touch upon topics that have natural connections with our constructions, like the construction of spreads of pseudolines from flat projective planes, the extendability of partial spherical circle planes to spherical circle planes and giving examples of sets of 2-arcs that determine flat projective planes and $\mathbb{R}^{2}$-planes.
\end{abstract}

\section{Spherical Circle planes}

1.1 Introduction. In this section we recall the definitions of some of the basic incidence geometric structures we shall be dealing with in the following. We shall also describe some of the fundamental relationships between them.

Let $P$ be a topological 2-sphere and let $\mathcal{K}$ be a set of simply closed curves on $P$. The pair $\mathcal{S}=(P, \mathcal{K})$ is called a spherical circle plane with point set $P$ and circle set $\mathcal{K}$ if it satisfies the axiom

(M1) Any three distinct points lie on a unique circle.

A spherical circle plane is called a flat Möbius plane if it satisfies the axiom

(M2) Given any circle $c$ and two distinct points $p$ and $q$ such that $p$ lies on $c$ and $q$ does not, there is a unique circle that contains both points and intersects $\boldsymbol{c}$ only in $\boldsymbol{p}$.

Examples of spherical circle planes arise as the geometries of non-trivial hyperplane sections of surfaces in $\mathbb{R}^{3}$ that are strictly convex and homeomorphic to the 2 -sphere. If the surface is differentiable, then every point of the surface is contained in a unique tangent hyperplane, that is, a hyperplane that intersects the surface only in this point. In this case the resulting spherical circle plane is a flat Möbius plane. The classical example of a flat Möbius plane is the circle plane associated with the unit sphere $\mathbb{S}^{2}$ in $\mathbb{R}^{3}$.

An $\mathbb{R}^{2}$-plane $(P, \mathcal{L})$ is a point-line geometry whose point set $P$ is a topological space homeomorphic to $\mathbb{R}^{2}$ and whose line set $\mathcal{L}$ consists of subsets of $P$ homeomorphic to

Received 15th June, 1995.

This research was supported by a Feodor Lynen fellowship.

Copyright Clearance Centre, Inc. Serial-fee code: 0004-9729/96 \$A2.00+0.00. 
$\mathbb{R}$ that separate $P$ into two open components. Furthermore, it satisfies the following axiom:

(A1) Two distinct points are contained in a unique line. An $\mathbb{R}^{2}$-plane is called a flat affine plane if it satisfies the axiom

(A2) Given a line $l$ and a point $p$, there is a unique line $l^{\prime}$ that contains $p$ and that is parallel to $l$, that is, $l$ does not intersect $l$ nor coincides with $l$.

The classical example of a flat affine plane is the Euclidean plane. The point-line geometry induced on any convex open subset of $\mathbb{R}^{2}$ yields an $\mathbb{R}^{2}$-plane.

A flat projective plane $(P, \mathcal{L})$ is a point-line geometry whose point set $P$ is a topological space homeomorphic to $\mathbb{P}^{2}$, the real projective plane (viewed as a topological space only). Its line set $\mathcal{L}$ consists of subsets of $P$ homeomorphic to the circle $\mathbb{S}^{1}$. Furthermore, it satisfies axiom $\mathrm{A} 1$ and the axiom

(A3) Two distinct lines intersect in a unique point.

The classical example of a flat projective plane is the real Desarguesian projective plane.

If one removes a line $W$ from a flat projective plane and punctures all other lines of the plane in the point of intersection with $W$, then the resulting point-line geometry is a flat affine plane. Furthermore, every flat affine plane $\mathcal{A}$ arises like this from a unique (up to isomorphism) flat projective plane. This flat projective plane is called the projective closure of $\mathcal{A}$ and $W$ the line at infinity of $\mathcal{A}$. The projective closure of the Euclidean plane is the real Desarguesian projective plane.

A 2-arc in an $\mathbb{R}^{2}$-plane or a flat projective plane $(P, \mathcal{L})$ is a subset of $P$ such that

(O1) every line in $\mathcal{L}$ intersects the set in no more than two points.

A 2-arc is a topological oval if it is homeomorphic to the unit circle and

(O2) every point of the 2-arc is contained in a unique tangent line, that is, a line that intersects the 2 -arc only in this point.

Examples of topological ovals in the Euclidean plane and its projective closure are the convex differentiable simply closed curves in $\mathbb{R}^{2}$.

Let $\mathcal{S}=(P, \mathcal{K})$ be a spherical circle plane, let $p$ be a point in $P$ and let $\mathcal{K}_{p}$ be the set of all circles in $\mathcal{K}$ that contain $p$ and that have been punctured at $p$. The pair $\mathcal{S}_{p}=\left(P \backslash\{p\}, \mathcal{K}_{p}\right)$ is an $\mathbb{R}^{2}$-plane. It is called the derived $\mathbb{R}^{2}$-plane of $\mathcal{S}$ at $p$. Every circle in $\mathcal{S}$ that does not contain $p$ is a 2 -arc in $\mathcal{S}_{p}$. If $\mathcal{S}$ is a flat Möbius plane, then the derived $\mathbb{R}^{2}$-plane $\mathcal{S}_{p}$ is a flat affine plane, the derived flat affine plane of $\mathcal{S}$ at $p$. Also, in this case, every circle in $S$ that does not contain $p$ is a topological oval in $\mathcal{S}_{p}$ and its projective closure $\bar{S}_{p}$.

All incidence geometries introduced so far are topological in the following sense: If $\mathcal{I}=(P, \mathcal{M})$ is such a geometry, then the set $\mathcal{M}$ carries a natural topology, the so 
called Hausdorff topology. With respect to this topology and the natural topology on the point set $P$ the geometric operations in the axioms that $\mathcal{I}$ satisfies are continuous. For example, if $\mathcal{I}$ is a spherical circle plane, then the connecting circle of three distinct points depends continuously on the positions of the three points in $P$.

The standard references for all the facts mentioned in this section are [16] for general spherical circle planes, [15] and [18] for flat Möbius planes, [12] and [13] for $\mathbb{R}^{2}$-planes and flat projective planes, $[1]$ for topological ovals, respectively.

1.2 REPRESENTATIONS OF FLAT PROJECTIVE PLANES ON DISKS, ARRANGEMENTS OF PSEUDOLINES AND SPREADS: Let $J$ be any simply closed curve in $\mathbb{R}^{2}$ and let $\gamma: J \rightarrow J$ be a continuous fixed-point-free involution. Then $J$ is the boundary of a topological compact disk $D_{J}$ and the topological space $D_{J} / \gamma$ we arrive at by identifying points on the boundary of this disk via $\gamma$ is homeomorphic to the real projective plane. Given any flat projective plane $\mathcal{P}=(P, \mathcal{L})$ and a line $l \in \mathcal{L}$, we can think of $P$ as being constructed in this way such that under the identification via $\gamma, P \backslash l$ is identified with the interior of the disk and the line $l$ is covered twice by $J$. This gives a representation of $\mathcal{P}$ on $D_{J}$. In this representation every point $p \in P$ corresponds to a point in the interior of the disk if $p$ is not contained in $l$ and to a pair of points $q, \gamma(q)$ on $J$ if $p$ is contained in $l$. The line $l$ corresponds to $J$, so $l=J / \gamma$. Any other line $k$ in $\mathcal{L}$ corresponds to a Jordan arc (homeomorphic to a closed interval) that connects the two points on $J$ that correspond to the unique point of intersection of $l$ and $k$. A flat projective plane given like this on a compact disk $D_{J}$ in $\mathbb{R}^{2}$, or in the one-point compactification $\mathbb{S}^{2}$ of $\mathbb{R}^{2}$, is said to be represented on the disk $D_{J}$ (via the involution $\gamma$ ). In [4] Gans discusses one particular representation of the real Desarguesian projective plane. Here $J$ is the unit circle, $D_{J}$ is the unit disk and $\gamma$ is the antipodal map. The lines in the disk are the halves of all ellipses that touch the unit circle in antipodal points and the straight line segments that connect antipodal points (see Figure 1). For further examples of disk representations of flat projective planes the reader is referred to $[8]$ and $[14]$.

The complement of a simply closed curve in $D_{J} / \gamma$ is either connected or not connected. A Jordan arc (homeomorphic to a closed interval) whose interior is contained in the interior of $D_{J}$ and whose endpoints are contained in $J$ and are exchanged by $\gamma$ corresponds to a curve of the first kind. We call Jordan arcs like this pseudolines. Clearly, all lines other than $J / \gamma$ in a representation of a flat projective plane on $D_{J}$ are pseudolines. A set of pseudolines in which two pseudolines intersect in a unique point is called a pseudoline arrangement. Finite pseudoline arrangements are of fundamental importance in the theory of oriented matroids (see [2, Chapter 6] and [7]). A spread (of pseudolines) on $D_{J} / \gamma$ is an infinite pseudoline arrangement such that

(S1) Every point $p$ on $J$ is contained in exactly one pseudoline $l(p)$ in the 


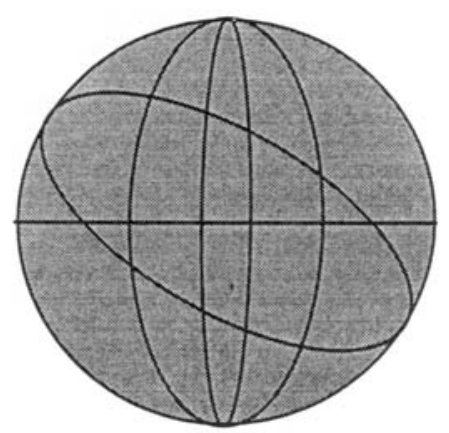

Figure 1.

arrangement.

(S2) The pseudoline $l(p)$ depends continuously on the point $p$ (in the Hausdorff metric).

An introduction to spreads can be found in [7, Chapter 4]. Every subset of lines excluding $J / \gamma$ of a flat projective plane represented on $D_{J}$ is a pseudoline arrangement. It has been shown in [8] that every finite pseudoline arrangement can be embedded as a subset of lines in a flat projective plane. A result about the extendability of finite pseudoline arrangements to spreads can be found in [9]. It is not known whether every spread of pseudolines can be embedded as a subset of lines in a flat projective plane.

Keeping in mind that flat projective planes are topological, it is clear how spreads can be constructed from a representation of a flat projective plane $\mathcal{P}$ on the disk $D_{J}$.

Proposition 1. Let $f:[0,1] \rightarrow J$ be an embedding such that $f(0)=\gamma(f(1))$ and let $h:[0,1] \rightarrow \operatorname{int}\left(D_{J}\right)$ be a continuous map such that $h(0)=h(1)$. Then the set of all connecting lines $f(t) \vee h(t), t \in[0,1)$ in the projective plane $\mathcal{P}$ is a spread of pseudolines in $D_{J} / \gamma$.

It is an open question whether all spreads arise from flat projective planes in this manner. As an example we construct a spread in the Gans representation (see Figure 1 ) by letting $h(t)$ equal the origin of the unit disk for all $t \in[0,1]$. Then, no matter how $f$ is chosen, the resulting spread consist of all straight line segments that connect antipodal points on the unit circle (see Figure 2.2).

Let $\mathcal{O}$ be a topological oval of $\mathcal{P}$ that is completely contained in the interior of $D_{J}$ (in [11] we proved that topological ovals exist in every flat projective plane). Let $\gamma^{\prime}$ be a fixed-point-free continuous involution of $\mathcal{O}$. Then we can construct spreads on the two disks $D_{J}$ and $D_{\mathcal{O}}$ as follows.

Proposition 2. Let $S$ be the set of all lines in $\mathcal{P}$ that contain pairs of points on $\mathcal{O}$ that get exchanged by $\gamma^{\prime}$. Then

(1) the set $S$ is a spread of pseudolines in $D_{J} / \gamma$; 


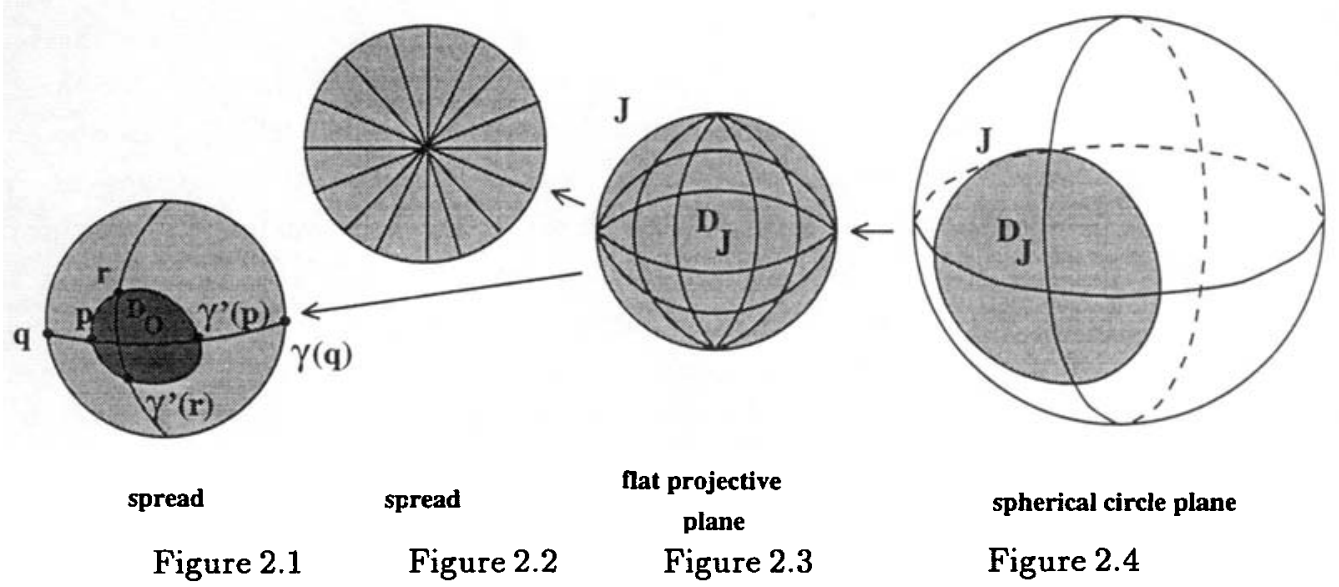

(2) the set of all intersections of elements in $S$ with $D_{\mathcal{O}}$ is a spread of pseudolines in $D_{\mathcal{O}} / \gamma^{\prime}$ (see Figure 2.1).

Proof: (1) Let $p, r \in \mathcal{O}$ such that $p \neq r, \gamma^{\prime}(r)$. Since the involution $\gamma^{\prime}$ is fixedpoint-free, $p$ and $\gamma^{\prime}(p)$ are contained in different components of $\mathcal{O} \backslash\left\{r, \gamma^{\prime}(r)\right\}$. This means that the elements of $S$ that contain the pairs $p, \gamma^{\prime}(p)$ and $r, \gamma^{\prime}(r)$ intersect somewhere in the disk $D_{\mathcal{O}}$. Axiom A3 implies that the two elements intersect $J$ in different pairs of points. Since $\mathcal{P}$ is topological it now follows easily that $S$ is a spread of pseudolines in $D_{J} / \gamma$.

(2) follows immediately from axiom $\mathrm{A} 1, \mathrm{O} 1$ and the fact that $\mathcal{P}$ is topological.

1.3 Constructing flat projective planes from SPherical Circle planes. In this section we describe how Figure 2.4 links up with Figures 2.1-2.3 by showing how flat projective planes represented on a disk can be constructed from spherical circle planes. The construction bears a strong resemblance to the construction of spreads described in Proposition 2(2).

Let $\mathcal{S}=(P, \mathcal{K})$ be a spherical circle plane and let $p$ be a point in $P$. Then $P \backslash\{p\}$ is homeomorphic to $\mathbb{R}^{2}$. If we identify both sets via some homeomorphism, for example, a stereographic projection, then the elements of $\mathcal{K}$ correspond to simply closed curves in $\mathbb{R}^{2}$ if they do not contain the point and to Jordan curves that separate $\mathbb{R}^{2}$ into two open components if they contain the point. Notice that the incidence structure whose point set is $\mathbb{R}^{2}$ and whose lines are the curves of the second kind is the derived $\mathbb{R}^{2}$-plane of the spherical circle plane $\mathcal{S}$ at $p$. For example, let $\mathcal{S}$ be the classical example of a flat Möbius plane, that is, the geometry of non-trivial hyperplane sections of the unit sphere $\mathbb{S}^{2}$ in $\mathbb{R}^{3}$. If we identify the unit sphere minus its "north pole $p$ " with $\mathbb{R}^{2}$ via the stereographic projection through $p$, then the circles that do not pass through $p$ are identified with the Euclidean circles in $\mathbb{R}^{2}$. The circles that 
pass through the point $p$ are identified with the straight lines in $\mathbb{R}^{2}$. Since the set of all straight lines in $\mathbb{R}^{2}$ makes up the line set of the Euclidean plane, we see that the derived flat affine plane of $\mathcal{S}$ at the point $p$ is isomorphic to the Euclidean plane. Of course, this is also true for any other point of $P$. By representing a spherical circle plane on $\mathbb{R}^{2}$ like this, no essential information about the circle plane gets lost since we can reconstruct the circle plane by one-point-compactifying $\mathbb{R}^{2}$ by some point $p$ and extending all Jordan arcs by this point (this makes them into subsets of the one-pointcompactification that are homeomorphic to the circle.) Therefore it makes sense to speak of a planar representation of a spherical circle plane. The pictures that we use in this section to illustrate various arguments always depict examples in the above planar representation of the classical spherical circle plane.

Let $\mathcal{S}=(P, \mathcal{K})$ be a spherical circle plane, let $J \in \mathcal{K}$ and let $\gamma: J \rightarrow J$ be a fixedpoint-free continuous involution. Let $D_{J}$ be one of the two compact disks bounded by $J$. Let $\mathcal{K}_{J, \gamma}$ be the set of all circles in $\mathcal{K}$ that contain one of the pairs of points $p$, $\gamma(p), p \in J$. Let $\mathcal{L}_{D_{J}, \gamma}$ be the set of intersections of elements in $\mathcal{K}_{J, \gamma}$ with $D_{J}$ and for any point $p \in P$ let $\mathcal{L}_{D_{J}, \gamma, p}$ be the set of all those elements in $\mathcal{L}_{D_{J}, \gamma}$ that come from circles that contain $p$. Finally, let $E_{D_{J}}$ be the set of all circles in $\mathcal{K}$ that are contained in the interior of $D_{J}$, let $P_{D_{J}}$ be the set of all circles in $\mathcal{K}$ that are contained in $D_{J}$ and that touch $J$ in exactly one point, and let $H_{D_{J}, \gamma}$ consist of all sets $D_{J} \cap k$ where $k \in \mathcal{K}$ intersects $J$ in two points that are not exchanged by $\gamma$.

We prove

Theorem 1. Let $\mathcal{S}=(P, \mathcal{K})$ be a spherical circle plane, $J \in \mathcal{K}, D_{J}$ one of the compact disks bounded by $J$ and $\gamma: J \rightarrow J$ a fixed-point-free continuous involution. Then

(1) $\mathcal{P}_{D_{J}, \gamma}=\left(D_{J}, \mathcal{L}_{D_{J}, \gamma}\right)$ is a flat projective plane represented on $D_{J}$.

(2) If $p \in P \backslash J$, then $\mathcal{L}_{D_{J}, \gamma, p}$ is a spread of pseudolines in $D_{J} / \gamma$.

(3) All elements of $E_{D_{J}}, P_{D_{J}}$ and $H_{D_{J}, \gamma}$ are 2-arcs in the flat projective plane $\mathcal{P}_{D_{J}, \gamma}$.

If $\mathcal{S}$ is a flat Möbius plane, then

(4) all elements of $E_{D_{J}}$ and $P_{D_{J}}$ are topological ovals in the flat projective plane $\mathcal{P}_{D_{J}, \boldsymbol{\gamma}}$.

ProOF: We mention that in a spherical circle plane two circles that intersect each other in two points intersect each other transversally, that is, they don't just touch in the points of intersection. We shall refer to the elements of $\mathcal{L}_{D_{J}, \gamma}$ as lines.

(2) Let $p \in P \backslash J$ and $q \in J$. By axiom M1, there is exactly one circle in $\mathcal{K}$ that contains the points $p, q$ and $\gamma(q)$. This circle corresponds to an element $l(q)$ in $\mathcal{L}_{D, \gamma, p}$. Let $r \in J$ be distinct from $q$ and $\gamma(q)$. Then, because $\gamma$ is fixed-point-free, 
the points $q$ and $\gamma(q)$ are contained in different connected components of $J \backslash\{r, \gamma(r)\}$. Hence two different lines in $\mathcal{L}_{D_{J}, \gamma, p}$ intersect in exactly one point in the interior of the disk $D_{J}$. Since $\mathcal{S}$ is topological, the line $l(q)$ depends continuously on $q$. Hence $\mathcal{L}_{D_{J}, \gamma, p}$ is a spread of pseudolines in $D_{J} / \gamma$.

(1) By $\left[12\right.$, Theorem 2.5], we only have to prove that $\mathcal{P}_{D_{J}, \gamma}$ satisfies axiom A1. Clearly, $J / \gamma$ is the only line that contains any two points contained in $J / \gamma$. Let $p$ be an interior point of $D_{J}$. By (2), we know that the set of lines containing $p$ is a spread and that any two lines in this set meet only in the point $p$. This implies that every point on $J$ is contained in precisely one line through $p$ and, by [7, Theorem 4.1], that every interior point different from $p$ is also contained in precisely one such line. This shows that $\mathcal{P}_{D_{J}, \gamma}$ satisfies axiom A1.

(3) This is an immediate consequence of axiom M1.

(4) Let $k$ be one of the circles under consideration. By (3) it satisfies axiom 01. Let $p \in k$ be one of the points in the interior of $D_{J}$. We need to show that there is exactly one tangent line in this point. The set $C$ of circles in $\mathcal{K}$ that touch $k$ in the point $p$ define a continuous involution $\gamma^{\prime}$ of $J$ as follows (see Figure 3.1 for the case that $k \in E_{D_{J}}$ and Figure 3.2 for the case that $k \in P_{D_{J}}$ ): Let $q$ be a point on $J$ and let $l$ be the unique circle in $C$ that contains $q$ (by M2). Then $l$ either touches $J$ in $q$ or it intersects $J$ in a second point. In the first case let $\gamma^{\prime}(q)=q$. In the second case let $\gamma^{\prime}(q)$ be the second point of intersection.

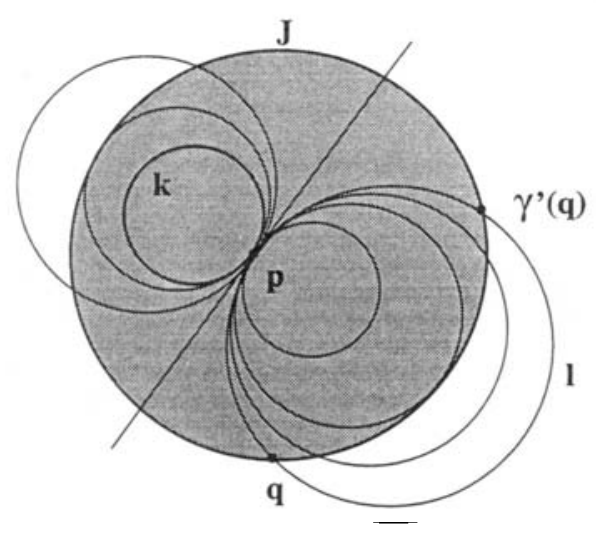

Figure 3.1

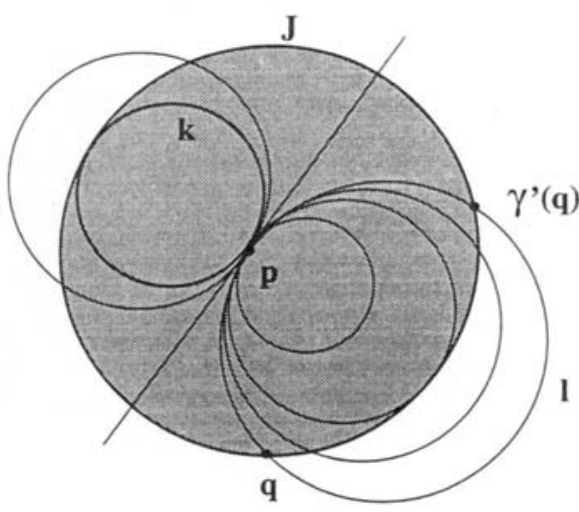

Figure 3.2

This involution has necessarily two fixed points (corresponding to the two circles in $C$ that touch $J$ ). Since $\gamma$ does not fix any point, by [17, Proposition 3.2a], there is exactly one pair of points $q, \gamma(q)$ on $J$ that is also exchanged by $\gamma^{\prime}$. The line in $\mathcal{L}_{D_{J}, \gamma}$ that corresponds to the circle in $C$ through these two points is a tangent line of the 2 -arc $k$ in this point. This tangent is unique since all lines through $p$ that do not arise 
from circles in $C$ intersect $k$ in a second point. If $k \in E_{D_{J}}$, every one of its points is an interior point of $D_{J}$, hence it satisfies axiom $\mathrm{O} 2$ and is therefore a topological oval. Let $p \in k$ be the unique point in which an element $k \in P_{D_{J}}$ touches $J$. Then $J / \gamma$ is clearly the unique tangent in this point. Again we conclude that $k$ is a topological oval.

CONSTRUCTING NON-ISOMORPHIC FLAT PROJECTIVE PLANES FROM THE CLASSICAL FLAT MöbIUS PLANE. Here is my favorite representation of the real Desarguesian projective plane on a disk: Let $J$ be the circle in the usual planar representation of the classical spherical circle plane that corresponds to the unit circle in $\mathbb{R}^{2}$, let $D_{J}$ be the unit disk and let $\gamma$ be the antipodal map. Then the lines in the flat projective plane $\mathcal{P}_{D_{J}, \gamma}$ are all the Euclidean line and circle segments that connect antipodal points (see Figure 4.1). It is easy to see that we are really dealing with a representation of the real Desarguesian projective plane. Just remember that in one of the most popular constructions of this plane we identify antipodal points of the unit sphere $\mathbb{S}^{2}$ in $\mathbb{R}^{3}$. This gives the point set of the plane. The lines are the images of the great circles on $\mathbb{S}^{2}$ under this identification. So a disk representation of the plane on the unit sphere is the restriction of the geometry of great circles to the "southern hemisphere". Our flat projective plane $\mathcal{P}_{D_{J}, \gamma}$ is the stereographic projection of this geometry on the $x y$-plane.

Figure 4.2 shows a Pappus configuration in this particular representation. We leave $D_{J}$ unchanged and choose $\gamma^{\prime}$ to be a continuous involution of $J$ that does exchange all the endpoints of the lines in Figure 4.2 except the endpoints $p$ and $\gamma(p)$ of the dashed line. This means that all of the configuration, except the dashed line will be contained in the flat projective plane $\mathcal{P}_{D_{j}, \gamma^{\prime}}$. Clearly, no line in this plane contains the three points $r, s$ and $t$. Hence $\mathcal{P}_{D_{J}, \gamma^{\prime}}$ is not Desarguesian, which means that our construction really yields non-isomorphic planes.

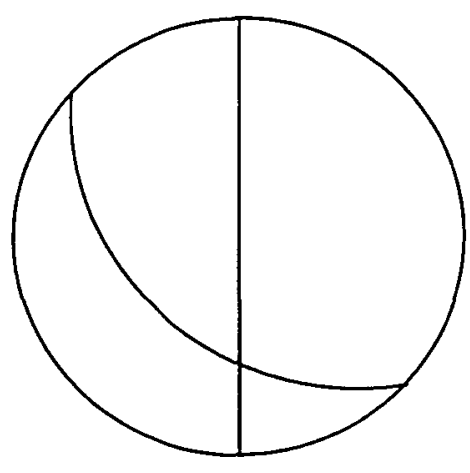

Figure 4.1

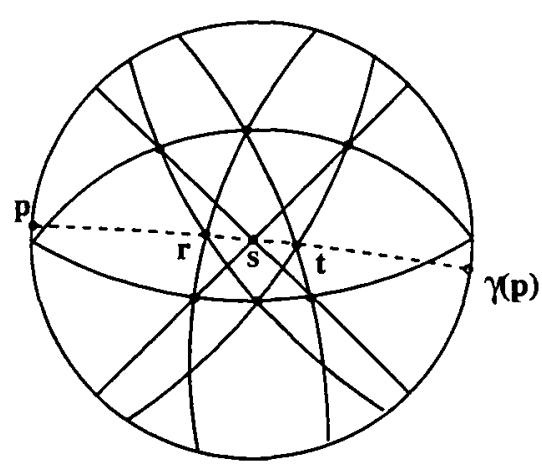

Figure 4.2

We also want to remark that special properties of the spreads in this representation 
of the real Desarguesian projective plane have been investigated in [19].

The Euclidean plane is orthogonal to the hyperbolic plane. Let $\mathcal{A}_{D_{J}, \gamma}$ be the flat affine plane we arrive at by removing the line $J / \gamma$ from the real Desarguesian projective plane $\mathcal{P}_{D_{J}, \gamma}$ (defined as in the previous remark). This particular representation of the Euclidean plane is remarkable insofar as it is 'orthogonal' to the Poincare model of the real hyperbolic plane (a special kind of $\mathbb{R}^{2}$-plane). We need to explain this idea: Figure 5.1 shows a parallel class of lines in this affine plane. Figure 5.2 depicts the uniquely determined set of Jordan arcs in the open unit disk that are orthogonal to all lines in the parallel class.

If we rotate both pictures at the same time around the origin we sweep aross all parallel classes and lines in the affine plane in Figure 5.1 and all lines in the Poincare model of the hyperbolic plane in Figure 5.2. In this way we get a partition of the line set of each plane into 'parallel classes' of lines and a one-to-one correspondence of the elements of these partitions such that corresponding elements are 'orthogonal'. We shall explore the concept of orthogonality, or even more generally of transversality, of $\mathbb{R}^{2}$-planes in a separate paper.

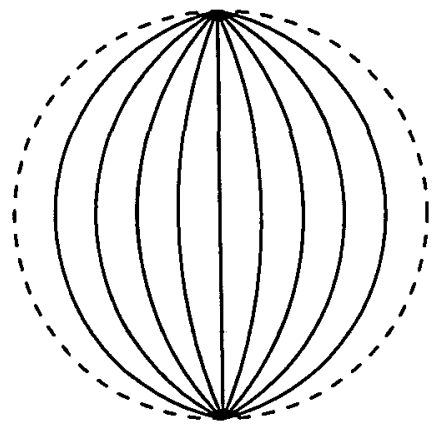

Figure 5.1

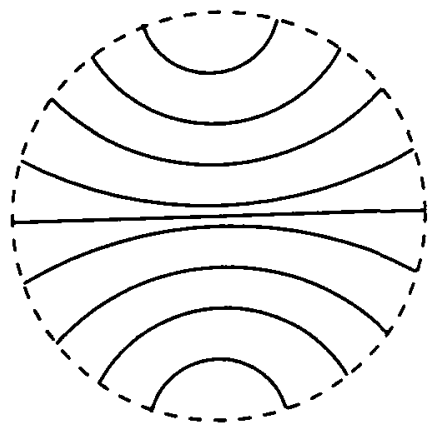

Figure 5.2

The Derived $\mathbb{R}^{2}$-Plane in a point as The Limit of Flat projective planes REPRESENTED ON EXPANDING DISKS. Let $S=(P, \mathcal{K})$ be a spherical circle plane. A circle $J$ in $\mathcal{K}$ bounds two compact disks on $P$. If we let $J$ shrink to a point $p$, then one of the disks gets arbitrarily small and the other one tends to fill out the whole of $P$ minus this point. Let continuous fixed-point-free involutions be defined (maybe in a continuous manner) on this shrinking $J$. If we don't worry too much about the details, then we get a picture of the flat projective plane $\mathcal{P}_{D_{J, \gamma}}$ on the disk $D_{J}$ that gets bigger tending to the derived flat affine plane at the point $p$.

Partial spherical circle planes. Let $\mathcal{S}=(P, \mathcal{K})$ be a spherical circle plane, let $\mathcal{P}_{D_{J}, \gamma}$ be constructed as in Theorem 1 and let $\mathcal{A}_{D_{J}, \gamma}$ be the flat affine plane we arrive 
at by removing the line $J / \gamma$ from the flat projective plane $\mathcal{P}_{D_{J, \gamma}}$. All the elments of $E_{D_{J}}$ are 2-arcs in this flat affine plane and $\mathcal{A}_{D_{J}, \gamma}$ together with this set of 2-arcs almost looks like the planar representation of a flat spherical circle plane. Of course, it is not, because there are triples of points of this geometry that get connected by, for example, elements of $P_{D_{J}}$ in the original circle plane $\mathcal{S}$. Still, it seems worthwhile to ask the question, whether this partial geometry can be made into a planar representation of a flat spherical plane by adding some more simply closed curves to its circle set. The answer to this question is 'No'. We can convince ourselves of this fact as follows: We can easily find a sequence of triples of distinct points that correspond to connecting circles in $E_{D_{J}}$ that converge to a triple of distinct points that corresponds to a connecting circle $k$ in $\boldsymbol{P}_{D_{J}}$. Because the spherical circle plane we are looking for would be topological, its planar representation would have to contain the intersection of $k$ with the interior of $D_{J}$. The resulting curve would be a Jordan arc, but all Jordan arcs have already been accounted for by the lines in the flat affine plane $\mathcal{A}_{D_{J}, \gamma}$.

Sets of 2-ARCS that Determine an $\mathbb{R}^{2}$-Plane. Let $\mathcal{S}=(P, \mathcal{K})$ be a spherical circle plane and let $D_{J}, J \in \mathcal{K}$ be a fixed compact disk in $P$. For different choices of the involution $\gamma$ we get different flat projective planes represented on this disk. Nevertheless all these planes share a large set of 2-arcs, namely $E_{D_{J}} \cup P_{D_{J}} \cup H_{D_{J}, \gamma}$. A natural question to ask is how 'large' a set of 2 -arcs in an $\mathbb{R}^{2}$-plane has to be to determine this $\mathbb{R}^{2}$-plane uniquely. One criterion is the following:

Proposition 3. Let $M$ be a set of 2 -arcs in an $\mathbb{R}^{2}$-plane or flat projective plane $\mathcal{P}=(P, \mathcal{L})$. If every three distinct points that are not contained in a line in $\mathcal{L}$ are contained in one of the 2-arcs in $M$, then $\mathcal{P}$ is the only $\mathbb{R}^{2}$-plane (flat projective plane) with point set $P$ in which every element of $M$ is a 2 -arc.

It is easy to see why this is true: Let $l$ be some line in $\mathcal{L}$ and let $p$ and $q$ be two points on $l$. The 2 -arcs in $M$ that contain these two points cover all the points of $P \backslash(l \backslash\{p, q\})$. Hence $l$ has to be contained in any $\mathbb{R}^{2}$-plane with point set $P$ in which every element of $M$ is a 2 -arc. Hence any such $\mathbb{R}^{2}$-plane contains all the lines of $\mathcal{P}$, which of course implies that $\mathcal{P}$ is the only such plane.

Let $\mathcal{P}$ be an $\mathbb{R}^{2}$-plane or a flat projective plane. Using the construction in [11], it is possible to construct a topological oval in $\mathcal{P}$ through any three given points of the plane that are not contained in a line in $\mathcal{P}$. This shows that

CoRollary 1. The set of topological ovals in an $\mathbb{R}^{2}$-plane or a flat projective plane determines the plane.

Also

COROLLARY 2. If $\mathcal{S}=(P, \mathcal{K})$ is a spherical circle plane and $p$ a point in $P$, then the set of all circles in $\mathcal{K}$ that do not pass through $p$ determine the derived $\mathbb{R}^{2}$-plane 
of $\mathcal{S}$ in the point $p$.

\section{Cylinder CiRCle Planes}

2.1 Introduction. A cylinder circle plane $\mathcal{S}=(P, \mathcal{K}, \|)$ consists of a point set $P$ which is the cylinder $\mathbb{S}^{1} \times \mathbb{R}$, a circle set $\mathcal{K}$ whose elements are graphs of continuous functions $\mathbb{S}^{1} \rightarrow \mathbb{R}$, and an equivalence relation $\|$ on the point set, the equivalence classes (parallel classes) of which are the verticals in $\mathbb{S}^{1} \times \mathbb{R}$, that is, the sets $\{(a, y) \mid y \in \mathbb{R}\}$, $a \in \mathbb{S}^{1}$. Furthermore, the incidence structure has to satisfy the axiom

(L1) Three pairwise non-parallel points are contained in a uniquely determined circle.

A cylinder circle plane is called a flat Laguerre plane if it satisfies the axiom

(L2) For two non-parallel points $p, q$ and a circle $c$ through $p$ there exists a uniquely determined circle through $q$ that touches $c$ at $p$, that is, intersects $c$ only in the point $p$, or coincides with $c$.

Examples of cylinder circle planes are (via some homeomorphism of the point sets onto the cylinder $\mathbb{S}^{1} \times \mathbb{R}$ ) the geometries of non-vertical hyperplane sections of a vertical cylinder in $\mathbb{R}^{3}$ over a strictly convex simply closed curve in the $x y$-plane. If the curve is differentiable, the corresponding cylinder circle plane is a flat Laguerre plane.

Like spherical circle planes, cylinder circle planes are topological: The common point set $\mathbb{S}^{1} \times \mathbb{R}$ of cylinder circle planes is a metrisable 2-dimensional topological space. Circles are homeomorphic to the unit circle $\mathbb{S}^{1}$. When the circle sets are topologised by the Hausdorff metric with respect to a metric that induces the topology of the point set, then the operations of joining three points by a circle and, in the case of flat Laguerre planes, touching are continuous. This has been proved for flat Laguerre planes by Groh (see $[\mathbf{6}, 3.10])$. For general cylinder circle planes this is a consequence of $[\mathbf{3}$, Theorem $1]$.

The standard references for flat Laguerre planes are [5, 6] and [15]. General cylinder circle planes have been investigated in the literature in the guise of periodic 3-unisolvent.sets (see $[3,10])$.

2.2 Constructing flat PROJeCtive Planes From Cylinder CirCle Planes. Let $\mathcal{S}=(P, \mathcal{K}, \|)$ be a cylinder circle plane, let $J \in \mathcal{K}$ and let $\gamma: J \rightarrow J$ be a fixed-pointfree continuous involution. We 2-point compactify the cylinder by two points $p_{u}, p_{d}$ (one for each of the two open ends). This 2-point compactification $\bar{P}$ is a topological space homeomorphic to the 2 -sphere. Let $D_{J}$ be one of the two compact disks on $\bar{P}$ bounded by $J$. The involution $\gamma$ induces an involution $\gamma_{\|}$on the set of parallel classes. If $p$ is the point of intersection of a vertical $v$ with $J$, then $\gamma_{\|}(v)$ is the vertical through the point $\gamma(p)$. Now, for every vertical $v$, we define a vertical circle that is the union 
of $v, \gamma_{\|}(v)$ and $\left\{p_{u}, p_{d}\right\}$. As a subset of $\bar{P}$ such a vertical circle is homeomorphic to the unit circle $\mathbb{S}^{1}$. Let $\overline{\mathcal{K}}$ be the union of $\mathcal{K}$ and the set of vertical circles. Let $\overline{\mathcal{K}}_{J, \gamma}$ be the set of all circles in $\overline{\mathcal{K}}$ that contain one of the pairs of points $p, \gamma(p), p \in J$. Notice that all vertical circles are contained in this set. Let $\mathcal{L}_{D_{J}, \gamma}$ be the set of intersections of elements in $\overline{\mathcal{K}}_{D_{J, \gamma}}$ with $D_{J}$, and for any point $p \in P$ let $\mathcal{L}_{D_{J}, \gamma, p}$ be the set of all those elements in $\mathcal{L}_{D_{J}, \gamma}$ that come from circles in $\overline{\mathcal{K}}$ that contain $p$. Finally, let $E_{D_{J}}$ be the set of all circles in $\mathcal{K}$ that are contained in the interior of $D_{J}$, let $P_{D_{J}}$ be the set of all circles in $\mathcal{K}$ that are contained in $D_{J}$ and that touch $J$ in exactly one point, and let $H_{D_{J}, \gamma}$ consist of all sets $D_{J} \cap k$ where $k \in \mathcal{K}$ intersects $J$ in two points that are not exchanged by $\gamma$.

We prove

TheOREM 2. Let $\mathcal{S}=(P, \mathcal{K}, \|)$ be a cylinder circle plane, $J \in \mathcal{K}, D_{J}$ be one of the compact disks in $\bar{P}$ bounded by $J$ and $\gamma: J \rightarrow J$ be a fixed-point-free continuous involution. Then:

(1) $\mathcal{P}_{D_{J}, \gamma}=\left(D_{J}, \mathcal{L}_{D_{J}, \gamma}\right)$ is a flat projective plane represented on $D_{J}$.

(2) If $p \in \bar{P} \backslash J$, then $\mathcal{L}_{D_{J}, \gamma, p}$ is a spread of pseudolines in $D_{J} / \gamma$.

(3) All elements of $E_{D_{J}}, P_{D_{J}}$ and $H_{D_{J}, \gamma}$ are 2-arcs in the flat projective plane $\mathcal{P}_{D_{j}, \gamma}$.

Let $\mathcal{S}$ be a flat Laguerre plane. Then:

(4) All elements of $E_{D_{J}}$ and $P_{D_{J}}$ are topological ovals in the flat projective plane $\mathcal{P}_{D_{J}, \gamma}$.

Proof: The disk $D_{J}$ contains only one of the points $p_{u}$ and $p_{d}$. We may assume that it contains $p_{u}$. To be able to illustrate some of our arguments, we want to think of the cylinder plus the point $p_{u}$ as being identified with $\mathbb{R}^{2}$ such that $p_{u}$ becomes the origin, and the verticals on the cylinder become the rays emanating from the origin. In this representation every circle in $\mathcal{K}$ turns into a simply closed curve that has the origin in its interior and that intersects every single one of the rays exactly once (see Figure 6.1).

Essentially, the same arguments as in the proof of Theorem 1 can be used to prove Theorem 2. We only sketch how the different arguments have to be modified. As in the case of spherical circle planes, two circles in a cylinder circle plane that intersect each other in two points intersect each other transversally, they don't just touch in the points of intersection.

(2) If $p$ is either $p_{u}$ or $p_{d}$ this is clearly the case. Let $p \in P \backslash J$ and $q \in J$. By axiom L1, there is exactly one circle in $\mathcal{K}$ that contains the points $p, q$ and $\gamma(q)$ if neither $q$ nor $\gamma(q)$ is parallel to $p$. If either $q$ or $\gamma(q)$ is parallel to $p$, then the vertical circle through $p$ contains all three points. So, there is a unique connecting circle of the three 


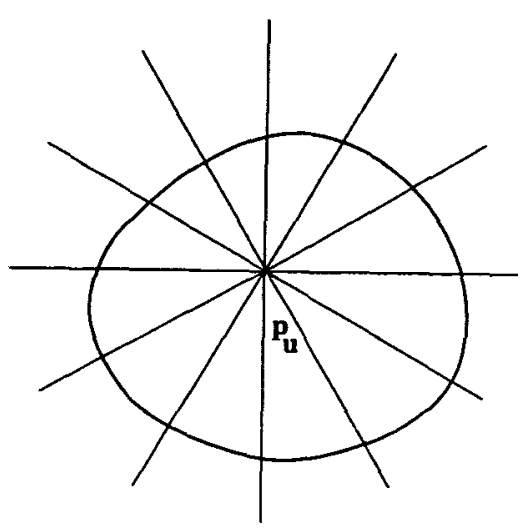

Figure 6.1

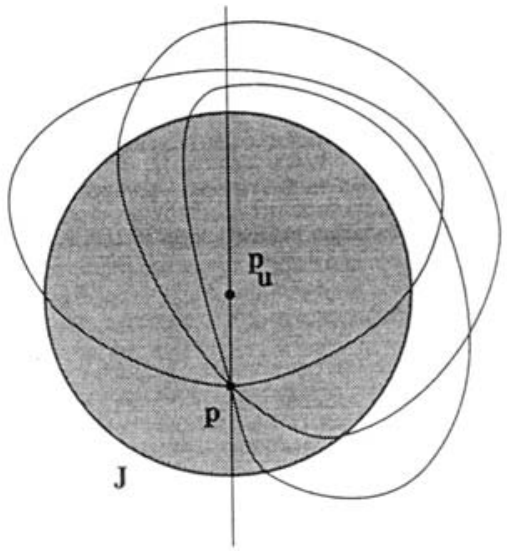

Figure 6.2

points in $\overline{\mathcal{K}}$. This circle corresponds to an element $l(q)$ in $\mathcal{L}_{D_{J}, \gamma, p}$. Two different liues in $\mathcal{L}_{D_{J}, \gamma, p}$ intersect in exactly one point in the interior of the disk $D_{J}$. Furthermore, $l(q)$ depends continuously on $q$. This follows from the fact that $\mathcal{S}$ is topological and the fact that $l(q)$ tends to the line that corresponds to the vertical circle through $p$ as $q$ (or $\gamma(q)$ ) tends to the point of intersection of the vertical circle through $p$ with $J$. This last fact is a consequence of a certain extension of the continuity of connecting points by circles, usually referred to as coherence (see [6, Paragraph 3]. Actually, Groh proves coherence of cylinder circle planes only in the case of flat Laguerre planes, but his proof carries over to general cylinder circle planes). Summarising, we conclude that $\mathcal{L}_{D_{J}, \gamma, p}$ is a spread of pseudolines in $D_{J} / \gamma$.

(1) We use the same argument as in the proof of Theorem 1 to prove this fact.

(3) This is an immediate consequence of axiom L1.

(4) Note that $p_{u}$ is never contained in any of the circles under consideration. Here we only have to modify the construction of the involution $\gamma^{\prime}$ of $J$ as follows (see Figure 7): Let $C$ be the set of all circles that touch $k$ in $p$. Let $q$ be a point on $J$ that is not parallel to $p$ and let $l$ be the unique circle in $C$ that contains $q$ (by L2). Then $l$ either touches $J$ in $q$ or it intersects $J$ in a second point. In the first case, let $\gamma^{\prime}(q)=q$. In the second case, let $\gamma^{\prime}(q)$ be the second point of intersection. If $q$ is the unique point on $J$ parallel to $p$, then $q$ is fixed by $\gamma^{\prime}$. That $\gamma^{\prime}$ is really continuous follows from the fact that in flat Laguerre planes touching is a continuous operation.

Similar remarks as at the end of Section 1 can be made at this point. Here are just two examples.

As in the spherical case it is possible to construct the real Desarguesian plane from the classical flat Laguerre plane as described in Theorem 2: Remember that this flat Laguerre plane is the geometry of non-trivial hyperplane sections of the vertical cylinder over the unit circle in the $x y$-plane. In particular, this unit circle is also a 


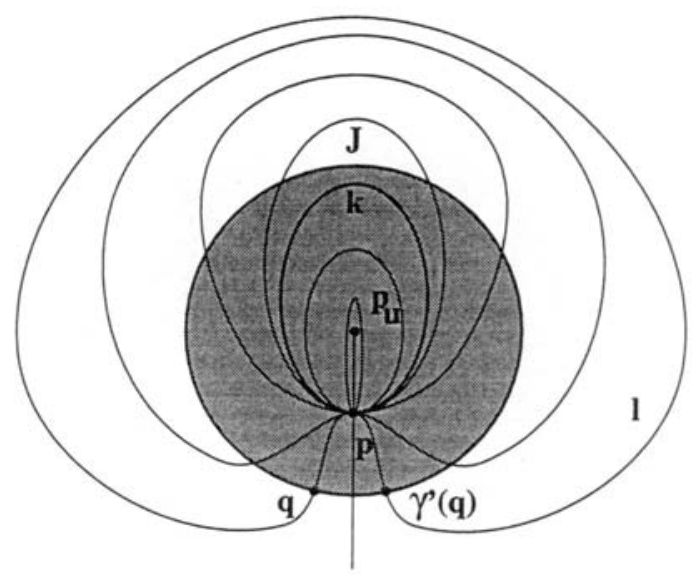

Figure 7

circle in the Laguerre plane and we choose it to be $J$. We choose the involution $\gamma$ to be the antipodal map of this unit circle. Then $\mathcal{P}_{D_{J, r}}$ is easily seen to be the real Desarguesian projective plane. Using the same idea as in Section 1 we can then leave $J$ unchanged and distort $\gamma$ slightly to arrive at a non-Desarguesian projective plane.

Planar representations of partial spherical circle planes can be constructed in very much the same manner as in Section 1. Using similar arguments as there, it can also be shown that these partial planes cannot be extended to planar representations of spherical circle planes.

2.3 Pairs of verticals as circles. A vertical cylinder in $\mathbb{R}^{3}$ with the unit circle in the $x y$-plane as a base can be considered as the limit of the family of ellipsoids $\left\{(x, y, z) \in \mathbb{R}^{3} \mid x^{2}+y^{2}+a z^{2}=1\right\}$ as $a$ tends to 0 . The geometries of non-trivial hyperplane sections of the ellipsoids give examples of flat Möbius planes. The geometry of non-vertical hyperplane sections of the cylinder is the classical model of a flat Laguerre plane. If a vertical hyperplane intersects any of the ellipsoids non-trivially, it intersects the cylinder in a pair of vertical lines. This suggests that a pair of verticals in a cylinder circle plane can play a similar role as a circle in the construction described above. This is indeed the case, as we shall see.

Let $\mathcal{S}=(P, \mathcal{K}, \|)$ be a cylinder circle plane, let $\{a\} \times \mathbb{R}$ and $\{b\} \times \mathbb{R}, a, b \in \mathbb{S}^{1}$ be two distinct verticals in $P$ and let $V$ be one of the two closed vertical strips bounded by the two verticals. The 2-point compactification of $V$ by the two points $p_{u}$ and $p_{d}$ is a compact disk $D_{a, b}$ with boundary $J_{a, b}=(\{a\} \times \mathbb{R}) \cup(\{b\} \times \mathbb{R}) \cup\left\{p_{u}, p_{d}\right\}$. A vertical in the interior of $V$ to which the two points $p_{u}$ and $p_{d}$ have been adjoined will be called a pseudovertical. Let $\gamma: J_{a, b} \rightarrow J_{a, b}$ be a fixed-point-free continuous involution 
with $\gamma\left(p_{u}\right)=p_{d}$. Let $\mathcal{K}_{J_{a, b}, \gamma}$ be the set of all circles in $\mathcal{K}$ that contain one of the pairs of points $p, \gamma(p), p \in J_{a, b}$. Let $\mathcal{L}_{D_{a, b}, \gamma}$ be the set that consists of all pseudoverticals, and the intersections of elements in $\mathcal{K}_{D_{a, b}, \gamma}$ with $D_{a, b}$.

TheOREM 3. Let $\mathcal{S}=(P, \mathcal{K}, \|)$ be a cylinder circle plane, and let $J_{a, b}, D_{a, b}$ and $\gamma: J_{a, b} \rightarrow J_{a, b}$ be defined as above. Then $\mathcal{P}_{D_{a, b}, \gamma}=\left(D_{a, b}, \mathcal{L}_{D_{a, b}, \gamma}\right)$ is a flat projective plane represented on $D_{a, b}$.

Essentially, a generalisation of this result to flat $n$-OAs has already been proven in [10, Proposition 2.5]. Nevertheless the general setting of this result is quite different from the one we use in this paper. We therefore include a sketch of a proof.

Proof: We call the elements of $\mathcal{L}_{D_{a, b}, \gamma}$ lines. By [12, Theorem 2.5], we only have to show that two distinct points $p$ and $q$ in $D_{a, b} / \gamma$ are connected by a unique line. If $p$ and $q$ are both contained in $J_{a, b} / \gamma$ or a pseudovertical, then $J_{a, b} / \gamma$ or this pseudovertical is clearly the only line containing both points. If $p$ is a point in the interior of $D_{a, b}$ and $q$ one of the points on $J_{a, b} / \gamma$, then L1 guarantees that there is a unique connecting line of the two points in $\mathcal{L}_{D_{a, b}, \gamma}$. Let $p$ and $q$ be interior points of $D_{a, b}$ that are not contained in the same pseudovertical and let $C$ be the set of circles in $\mathcal{K}$ that contain both points. We define a function $g: \mathbb{R} \rightarrow \mathbb{R}$ that maps $x$ to $y$ if and only if there is a circle in $C$ that intersects the verticals $\{a\} \times \mathbb{R}$ and $\{b\} \times \mathbb{R}$ in the points $(a, x)$ and $(b, y)$, respectively. Since $\mathcal{S}$ is a flat cylinder plane this map is an orientation-preserving homeomorphism. The involution $\gamma$ induces a orientation-reversing homeomorphism $h: \mathbb{R} \rightarrow \mathbb{R}$ as follows: The point $x$ is mapped to $y$ if and only if $\gamma((a, x))=(b, y)$. It is clear that there is exactly one $x_{0} \in \mathbb{R}$ such that $g\left(x_{0}\right)=h\left(x_{0}\right)$. Therefore the line through $\left(a, x_{0}\right), p$ and $\gamma\left(\left(a, x_{0}\right)\right)$ is the unique line in $\mathcal{L}_{D_{a, b}, \gamma}$ that connects the two points $p$ and $q$.

\section{REFERENCES}

[1] T. Buchanan, H. Hähl and R. Löwen, 'Topologische Ovale', Geom. Dedicata 9 (1980), 401-424.

[2] A. Björner, M. Las Vergnas, B. Sturmfels, N. White and G. Ziegler, Oriented matroids, Encyclopedia of Mathematics and its Applications 46, (Cambridge University press, Cambridge, 1993).

[3] Ph.C. Curtis, Jr., 'n-parameter families and best approximation', Pacific J. Math. 9 (1959), 1013-1027.

[4] D. Gans, 'A circular model of the euclidean plane', Amer. Math. Monthly 61 (1954), 23-30.

[5] H. Groh, 'Topologische Laguerreebenen I', Abh. Math. Sem. Univ. Hamburg 32 (1968), 216-231. 
[6] H. Groh, 'Topologische Laguerreebenen II', Abh. Math. Sem. Univ. Hamburg 34 (1970), 11-21.

[7] B. Grünbaum, Arrangements and spreads, CBMS Regional Conference Series in Math. 10 (American Mathematical Society, Providence, R.I, 1972).

[8] J.E. Goodman, R. Pollack, R. Wenger and T. Zamfirescu, 'Arrangements and topological planes', Amer. Math. Monthly 101 (1994), 866-877.

[9] J.E. Goodman, R. Pollack, R. Wenger and T. Zamfirescu, 'Every arrangement extends to a spread', Combinatorica 14 (1994), 301-306.

[10] B. Polster, 'n-unisolvent sets and flat incidence structures', (preprint).

[11] B. Polster, G.F. Steinke and N. Rosehr, 'On the existence of topological ovals in flat projective planes', (preprint).

[12] H. Salzmann, 'Topological planes', Adv. Math. 2 (1967), 1-60.

[13] H. Salzmann, D. Betten, T. Grundhöfer, H. Hähl, R. Löwen and M. Stroppel, Compact projective planes (de Gruyter, Berlin, 1995).

[14] M. Sholander, 'Plane geometries from convex plates', Pacific J. Math. 3 (1953), 667-671.

[15] G.F. Steinke, 'Topological circle geometries', in Handbook of Incidence Geometry, (F. Buekenhout, Editor) (Elsevier, 1995), pp. 1325-1353.

[16] K. Strambach, 'Sphärische Kreisebenen', Math. Z. 113 (1970), 266-292.

[17] G. Valette, 'Structures d'ovale topologique sur le cercle', Bull. Acad. R. Belg. 51 (1965), 586-597.

[18] R.D. Wölk, 'Topologische Möbiusebenen', Math. Z. 93 (1966), 311-333.

[19] T. Zamfirescu and A. Zucco, 'Continuous families of smooth curves and Grünbaum's conjecture', Canad. Math. Bull. 27 (1984), 345-350.

\footnotetext{
Department of Pure Mathematics

The University of Adelaide

Adelaide SA 5005

Australia

e-mail: bpolster@maths.adelaide.edu.au
} 